ReCAPs (Research

Contributions Abbreviated for Print) provide a structured, one-page summary of each paper highlighting the main findings and significance of the work. The full version of the article is available online at jop.ascopubs.org.

Magee-Women's Hospital of the University of Pittsburgh Medical Center; University of Pittsburgh Graduate School of Public Health; University of Pittsburgh, Pittsburgh, PA; University of Colorado Denver, Aurora, CO; Northwestern University, Chicago, IL; University of Alabama at Birmingham, Birmingham, AL; University of North Carolina, Chapel Hill, NC; University of Virginia School of Medicine, Charlottesville, VA; and University of Washington, Seattle, WA

Corresponding author: Casey M. Hay, MD, Division of Gynecologic Oncology,

Department of Obstetrics, Gynecology and Reproductive Sciences, Magee-Women's Hospital of the University of Pittsburgh Medical Center, 300 Halket St, Pittsburgh, PA 15213; e-mail: haycm2@upmc.edu.

Disclosures provided by the authors are available with this article at jop.ascopubs.org.

DOl: https://doi.org/10.1200/JOP. 2017.020818; published online ahead of print at jop.ascopubs.org on August 1, 2017.

\title{
Strategies for Introducing Outpatient Specialty Palliative Care in Gynecologic Oncology
}

\author{
Casey M. Hay, Carolyn Lefkowits, Megan Crowley-Matoka, Marie A. Bakitas, Leslie H. Clark, Linda R. Duska,
} Renata R. Urban, Stephanie L. Creasy, and Yael Schenker

QUESTION ASKED: How do gynecologic oncologists introduce patients to outpatient specialty palliative care?

SUMMARY ANSWER: Gynecologic oncologists at academic institutions with wellestablished palliative care services have developed several strategies for introducing patients to palliative care. These strategies aim to alleviate patient concerns and encourage acceptance of early palliative care referral.

WHAT WE DID: We conducted a national qualitative interview study at six academic cancer centers with well-established palliative care clinics. Thirty-four gynecologic oncologists participated in semistructured telephone interviews focusing on attitudes, experiences, and practices related to outpatient palliative care. A multidisciplinary team analyzed interview transcripts using constant comparative methods to inductively develop and refine a coding framework.

WHAT WE FOUND: Gynecologic oncologists described the following three main strategies for introducing outpatient specialty palliative care: focus initial palliative care referral on symptom management to dissociate palliative care from end-of-life care and facilitate early relationship building with palliative care clinicians; use a strong physician- patient relationship and patient trust to increase acceptance of referral; and explain and normalize palliative care referral to address negative associations and decrease patient fear of abandonment. These strategies aim to decrease negative patient associations and encourage acceptance of early referral to palliative care specialists.

BIAS, CONFOUNDING FACTOR(S), REALLIFE IMPLICATIONS: Strengths of our study include the large qualitative sample size and high response rate, as well as the inclusion of providers from multiple institutions with varying geography, size, and palliative care system structure. Because our study only included gynecologic oncologists at academic institutions with established outpatient palliative care clinics, our results may not be generalizable to medical, surgical, or radiation oncologists or to community practices or settings where palliative care is less available or less well established. In addition, the design of our study did not allow us to measure actual referral practices. These findings provide the groundwork for developing a system-wide best practice approach to introducing palliative care referral within gynecologic oncology that may help facilitate adherence to national recommendations for early initiation of palliative care. 


\section{Strategies for Introducing Outpatient Specialty Palliative Care in Gynecologic Oncology}

Casey M. Hay, Carolyn Lefkowits, Megan Crowley-Matoka, Marie A. Bakitas, Leslie H. Clark, Linda R. Duska, Renata R. Urban, Stephanie L. Creasy, and Yael Schenker

Magee-Women's Hospital of the University of Pittsburgh Medical Center; University of Pittsburgh Graduate School of Public Health; University of Pittsburgh, Pittsburgh, PA; University of Colorado Denver, Aurora, CO; Northwestern University, Chicago, IL; University of Alabama at Birmingham, Birmingham, AL; University of North Carolina, Chapel Hill, NC; University of Virginia School of Medicine, Charlottesville, VA; and University of Washington, Seattle, WA

\section{ASSOCIATED CONTENT}

See accompanying editorial on page 597

Data Supplement available online
DOl: https://doi.org/10.1200/JOP. 2017.020818; published online ahead of print at jop.ascopubs.org on August 1, 2017.

\section{Abstract}

\section{Purpose}

Concern that patients will react negatively to the idea of palliative care is cited as a barrier to timely referral. Strategies to successfully introduce specialty palliative care to patients have not been well described. We sought to understand how gynecologic oncologists introduce outpatient specialty palliative care.

\section{Methods}

We conducted a national qualitative interview study at six geographically diverse academic cancer centers with well-established palliative care clinics between September 2015 and March 2016. Thirty-four gynecologic oncologists participated in semistructured telephone interviews focusing on attitudes, experiences, and practices related to outpatient palliative care. A multidisciplinary team analyzed interview transcripts using constant comparative methods to inductively develop and refine a coding framework. This analysis focuses on practices for introducing palliative care.

\section{Results}

Mean participant age was 47 years (standard deviation, 10 years). Mean interview length was 25 minutes (standard deviation, 7 minutes). Gynecologic oncologists described the following three main strategies for introducing outpatient specialty palliative care: focus initial palliative care referral on symptom management to dissociate palliative care from end-of-life care and facilitate early relationship building with palliative care clinicians; use a strong physician-patient relationship and patient trust to increase acceptance of referral; and explain and normalize palliative care referral to address negative associations and decrease patient fear of abandonment. These strategies aim to decrease negative patient associations and encourage acceptance of early referral to palliative care specialists.

\section{Conclusion}

Gynecologic oncologists have developed strategies for introducing palliative care services to alleviate patient concerns. These strategies provide groundwork for developing systemwide best practice approaches to the presentation of palliative care referral.

\section{INTRODUCTION}

Early integration of specialty palliative care into standard oncology care is promoted by national organizations, including ASCO, the National Comprehensive Cancer Network, and the Society of
Gynecologic Oncology. ${ }^{1-3}$ Specialty palliative care improves quality of life and may increase survival among patients with advanced cancer. ${ }^{4-9}$ Timely palliative care referral is essential in providing quality cancer care. 
Despite its proven benefits, palliative care in oncology is still often delivered late in the disease course. ${ }^{10,11}$ Oncologist concern that patients will react negatively to the idea of palliative care has been frequently identified as a barrier to timely referral. ${ }^{12-17}$ This early barrier of patient decline of referral can severely limit the benefits reaped from integrated palliative care. As a result, understanding how oncologists effectively approach and address this concern would provide groundwork to guide future oncologist education and improve the integration of palliative care into standard oncology care.

We sought to understand how gynecologic oncologists introduce outpatient specialty palliative care and identify strategies used to facilitate patient acceptance of referral. We chose to examine the use of outpatient palliative care because outpatient referrals occur earlier in the disease course than inpatient consultations ${ }^{10}$ and are thus most concordant with recommendations for early palliative care referral.

\section{METHODS}

\section{Study Design}

We conducted a qualitative, in-depth interview study to understand gynecologic oncologists' views on outpatient specialty palliative care. This study was approved by the University of Pittsburgh Institutional Review Board (PRO15070206). Results from this study have been previously published ${ }^{18}$; however, no prior analysis has examined the strategies gynecologic oncologists use to present palliative care to their patients.

\section{Recruitment}

Gynecologic oncologists at six National Cancer Institutedesignated cancer centers with well-established outpatient palliative care clinics were approached for participation. Initial contact was by e-mail with follow-up to schedule interviews conducted via a combination of e-mail and direct contact by local colleagues. Institutions were selected from across the United States to ensure diversity of geography, gynecologic oncology program size, and palliative care clinic structure (Table 1). Eligible participants were attending-level, fellowship-trained gynecologic oncologists with an outpatient clinical practice of at least 1 halfday per week. Study authors were excluded from participation.

\section{Interviews}

Semistructured, in-depth interviews were conducted via phone by a single study investigator trained in semistructured interviewing techniques (C.M.H.). Interviews occurred between September 2015 and March 2016. The study was approved by the University of Pittsburgh Institutional Review Board, and all participants provided verbal informed consent. Standard in-depth interviewing techniques focused on oncologist experiences and practices surrounding referral to outpatient specialty palliative care. Questions were designed to be open-ended and nonleading, and follow-up probes were used to elicit rich description of individual experiences. The interviewer was trained to pose questions in a neutral and nondirective manner. Questions elicited reflection on real patients and also presented brief vignettes involving hypothetical patients. The full interview guide is available in the Data Supplement.

\section{Analysis}

Audio recordings of interviews were transcribed verbatim. Line-by-line coding of a subset of transcripts facilitated inductive development of an initial coding framework. This technique, known as qualitative description, describes events or phenomena and the meanings attributed to these by participants. ${ }^{19}$ Concepts and codes were discussed, compared, and refined using constant comparative methodology ${ }^{20}$ by a multidisciplinary team with expertise in palliative care, gynecologic oncology, qualitative research methodology, advanced practice nursing, anthropology, and public health. Emerging codes were iteratively applied to transcripts as they were obtained, with team discussion until agreement was reached on a final coding framework. The final coding framework was applied to all transcripts by a trained investigator (C.M.H.), and a second investigator (S.L.C.) coded $50 \%$ of the interviews, with comparison undertaken to ensure intercoder agreement. Disagreements were discussed and resolved by team consensus. Codes were classified, grouped, and refined into themes through an iterative, analytic process. ${ }^{19}$ ATLAS.ti Software (V 5.5.9; Scientific Software Development, Berlin, Germany) facilitated data analysis.

\section{RESULTS}

Thirty-four of 35 eligible gynecologic oncologists at six different institutions participated, for a response rate of $97 \%$. One physician was unable to participate because of scheduling conflicts. Participants were gynecologic oncology board certified or eligible. One participant was board certified in palliative care, and no participants had completed a palliative care fellowship. Table 2 lists clinician characteristics. Average 
Table 1. Institution Characteristics

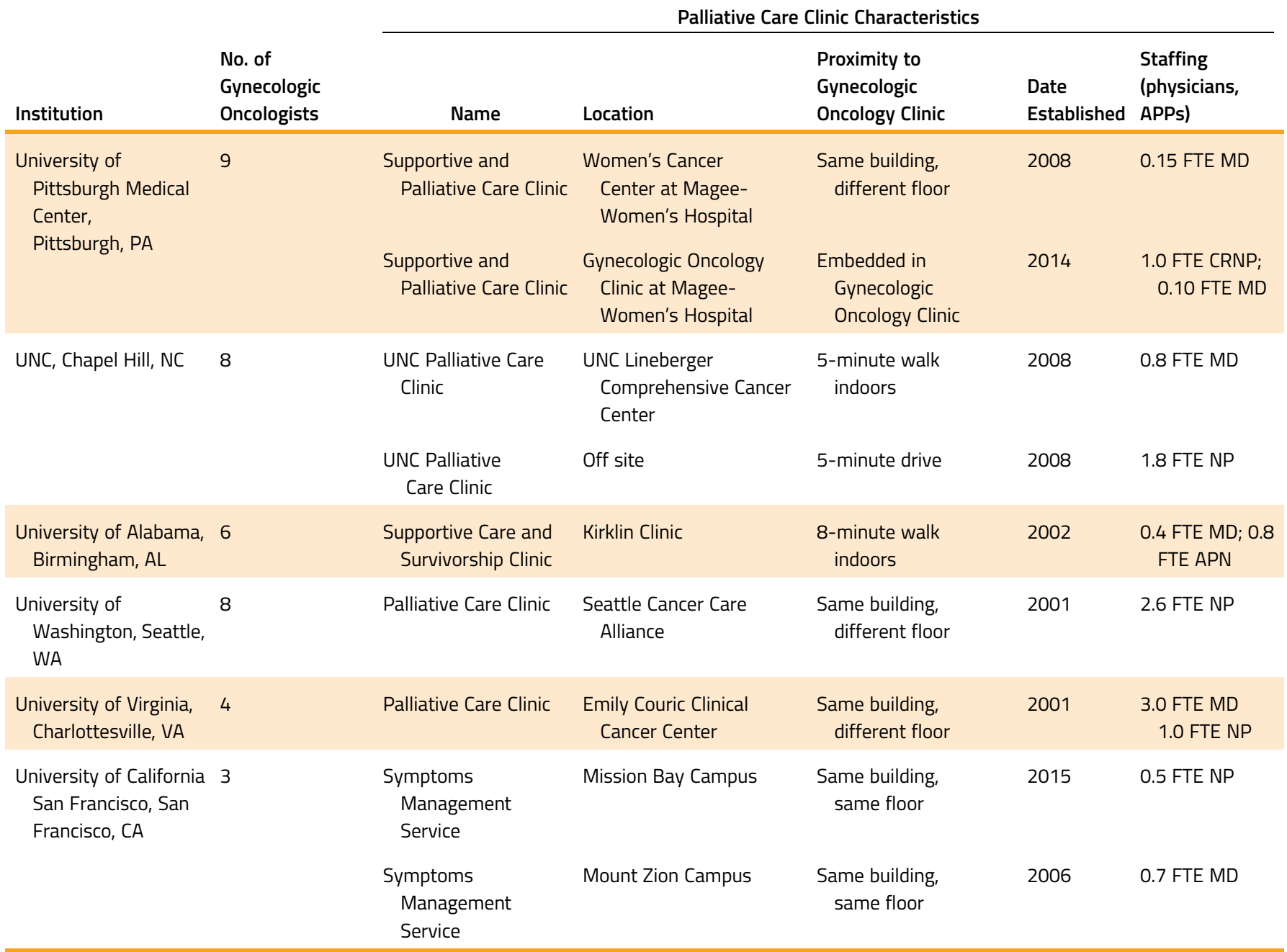

Abbreviations: APN, advanced practice nurse; APP, advanced practice provider; CRNP, certified registered nurse practitioner; FTE, full-time equivalent; MD, physician; NP, nurse practitioner; UNC, University of North Carolina.

interview length was 25 minutes (standard deviation, 7 minutes; range, 14 to 45 minutes).

In analysis of participant interviews, the following three themes emerged related to strategies gynecologic oncologists used to introduce outpatient specialty palliative care to their patients: focus initial palliative care referral on symptom management to dissociate palliative care from end-of-life care and facilitate early relationship building with palliative care clinicians; use a strong oncologistpatient relationship and patient trust to increase acceptance of referral; and normalize and explain palliative care referral to address negative associations and decrease patient fear of abandonment.

\section{Focus Initial Palliative Care Referral on Symptom Management to Dissociate Palliative Care From End- of-Life Care and Facilitate Early Relationship Building With Palliative Care Clinicians}

A majority of participants (20 of 34 participants) discussed using symptom management as the initial reason for referral to outpatient specialty palliative care to disassociate referral from end-of-life care (Table 3). As one participant described:

I try to kind of disconnect hospice from palliative care, you know, and so I think she seemed open to talking with them, especially when I focused on the ways that they could try and help her with the symptoms that she was experiencing. 
Table 2. Participant Characteristics

\begin{tabular}{lc} 
Characteristic & No. of Participants (\%) \\
\hline $\begin{array}{l}\text { Age, years } \\
\leq 35\end{array}$ & $3(9)$ \\
$36-45$ & $14(41)$ \\
$46-55$ & $11(32)$ \\
$56-65$ & $4(12)$ \\
$>65$ & $2(6)$ \\
\hline Sex & \\
Female & $19(56)$ \\
Male & $15(44)$ \\
\hline Race & \\
White & $23(67)$ \\
African American & $3(9)$ \\
Asian & $7(21)$ \\
Hispanic & $1(3)$ \\
\hline Time in practice since residency, years & \\
$<5$ & $4(12)$ \\
$5-10$ & $8(24)$ \\
$11-15$ & $7(20)$ \\
$16-20$ & $5(15)$ \\
$>20$ & $10(29)$ \\
\hline
\end{tabular}

Physicians anticipated that referral for reasons associated with end-of-life or hospice care would cause distress in their patients and be met with resistance or refusal and felt that reframing the referral in the context of symptoms fostered acceptance: “She was fine with it because it wasn't done in the context of 'this is headed towards death'; it was done in the context of she has symptoms related to her cancer." One participant explained how referring for symptom management facilitated both early referral and patient acceptance:

You get them involved before she starts to go down the drain so she doesn't feel like these people are being introduced to have the death and dying talk with her.... A lot of these patients their only association with palliative care equates to hospice, and so if you can introduce them before you start having those kind of discussions, the focus can then be on what she wants moving forward, how we're going to control her symptoms, and easing us into that conversation when the time comes

Referral for symptom management was also frequently identified as a mechanism to promote relationship building between palliative care clinicians and patients with poor prognoses. As one participant explained:
[There are] two benefits, there's short term, and short term is better management of her symptoms that affect her quality of life, but long term, to have people around who she becomes familiar with so when we're ready to have hospice she's comfortable with the decision because these aren't strangers to her.

This strategy was felt to facilitate earlier referral to palliative care and increase patient acceptance: "Earlier referral makes it not such a scary concept when you talk about it later; when the phases of care change, they're already comfortable with that, so I think that's one benefit." Another participant explained how this approach enabled him to provide an improved quality of care:

\begin{abstract}
The destiny for those patients after recurrence of fallopian or ovarian cancer is that as a rule they will ultimately die of that disease, so I like to get them involved early...so it's an ongoing relationship that's built over a period of time as opposed to the old practice of referring a patient to hospice care and they pass on within two to four weeks of a referral which is inferior care.
\end{abstract}

\section{Use a Strong Oncologist-Patient Relationship and Patient Trust to Increase Acceptance of Referral}

Many participants (16 of 34 participants) identified a strong relationship with their patients as key to patient acceptance of palliative care referral (Table 3). A strong oncologist-patient relationship was felt to facilitate open dialogue and allow the physician to tailor the timing and content of discussions about palliative care referral. One participant explained how the strength of her relationships with patients enabled her to more effectively initiate conversations about palliative care referral:

\begin{abstract}
Because we do the long-term follow-up for our patients and we know them so well, I think it in many ways it just makes it easier to have very frank conversations with patients about things like involvement of palliative care.
\end{abstract}

In addition to aiding the initiation of conversations about palliative care referral, strong oncologist-patient relationships were felt to help oncologists individualize the discussion and lead to increased acceptance:

Different patients and different families have different reactions to the words palliative care, so I think that it is important to know your patient, have a sense of how they're going to respond to that and I guess I would say frame it in the right way. 


\section{Table 3. Representative Quotations Supporting Identified Strategies}

\section{Strategy}

Focus initial palliative care referral on symptom management to dissociate palliative care from end-of-life care and facilitate early relationship building with palliative care clinicians

\section{Representative Quotations}

But I don't typically say "I'm gonna have you see hospice." I may say "I'm going to have you see someone from palliative care; the goal is really to alleviate some of your symptoms." That's how I sort of tell them what the referral is for.

She didn't have a lot of symptoms but had some pain issues so I referred her to supportive care to assist with kind of pain management and just to kind of introduce the idea of supportive care, given her stage IV diagnosis and kind of overall poor prognosis.

I felt like these were not going to be temporary issues and the likelihood of her having a complete resolution of her cancer in the near future was pretty small, so I wanted their assistance because I knew, not only for now, but the likelihood of her having to transition to a completely supportive care approach was going to be likely necessary.

I think I also...expect them to have a better transition into hospice.... I would expect that having palliative care on board, you know that that transition as things get worse, to being more weighted to doing symptom management versus disease management, will be smoother.

Use a strong oncologist-patient relationship and patient trust to increase acceptance of referral
I think [long-term relationships] allow you to have trust with your patients.... There's no question for my longer term patients or patients I've known a long time, not that all of them want the palliative care services, but you know I feel more comfortable when I bring it up; they're not as "What do you mean there's nothing left" or things like that because we've had discussions over the years.

We have a unique bond with our patients because we go through much more of their treatment with them...so I think we have a unique relationship with the patients where they really bond with us and that gives us the opportunity to really make sure that all of their needs are being met and to really make sure that they make sure that they get in to see the supportive care team.

These are often women who we have a long-standing relationship with, with both the patient and their families, and I would imagine that sometimes makes the introduction of palliative care, or the concept of palliative care, or hospice care, or addressing goals of care, actually a little bit easier.

I think there is a negative connotation with palliative care. I think a lot of people worry that if they sign up for that it means you're giving up on them [but] I think once you explain things people are a little more comfortable.

It's usually not an issue if it's introduced appropriately and as long as the indication is explained well by the referring physician. So as long as I do a good job of explaining why the patient would benefit from it, I feel like it's usually not an issue.... If the patient can't grasp the reasons why the palliative care service would be beneficial to them, I just worry that they're going to be like "Well why would I need to see them? Are you saying that there's no hope for me? Am I gonna die?" Like those kinds of things

After taking the time to talk to them about it, which is another problem, taking the time, because nobody has time, but if you talk to them about it and kind of dispel the negative connotation of palliative and telling them it's also supportive care and what their role can be in quality of life and things like that and telling them that it's literally just a change in the focus of their cancer care, most people will do it.
One participant discussed how the trust that develops from strong relationships promotes acceptance of referral:

I think you develop a trust with the patient and it's just another thing that you're recommending to them, and it's not seen as out of the blue, it's just part of the care.... I think if you're comfortable and they trust you, they're going to kind of accept and trust what you're recommending.

A strong oncologist-patient relationship was also felt to increase acceptance of palliative care referral by allowing a longitudinal discussion. As one participant explained, "If they seem a little bit resistant, I kind of address the need; we bring it up, we talk about it, and I try to circle back to it in the subsequent visits." The perceived value of a strong relationship in promoting acceptance was further illustrated by another participant who described waiting to make a palliative care referral until she developed a stronger relationship with her patients:

I might introduce the concept but not make the referral yet, just telling them that we work together, and it's somebody that they'll meet down the road, and then once we've established a treatment plan and they've met me, and started a relationship with me, then introducing it after a couple of visits. 


\section{Explain and Normalize Palliative Care Referral to Address Negative Associations and Decrease Patient Fear of Abandonment}

Many participants (16 of 34 participants) emphasized the importance of explaining and normalizing palliative care to increase patient acceptance of referral (Table 3). Participants frequently attributed patient resistance to the association of the term palliative care with hospice. Participants felt that this association could be overcome by taking the time to clearly explain palliative care. As one participant explained:

I think that it's all about the language. I can tell you, to the patient the word "palliative" is synonymous with hospice, is synonymous with "I'm dying and the doctor is now giving up" in the perception of the patient, but it depends on how it is introduced to them.

Another participant remarked:

\begin{abstract}
I think the problem is that palliative care has a horrible name...because it just brings up a lot of negative connotations to people, rightly or wrongly. I think a lot of people think when you say palliative care they almost equate that to hospice and giving up, and so I think once you explain that it really is more about aggressive symptom management and enhancement of quality of life, but I think for the most part it always requires a little bit of effort to explain what palliative care does.
\end{abstract}

Patient fear of abandonment was also frequently discussed as a reason for resistance to referral, and participants felt that this also could be addressed with time and clear explanation. One participant explained how upfront discussion could prevent a patient from feeling abandoned:

I think there's always the concern that the patient feels like you're writing them off as a lost case, that you're losing hope in them, and that you're giving up on them, but if that is addressed at the outset and that discussion is hadand it's not just "Here, go see these people" and it's not introduced appropriately then it's a problem, but as long as it's introduced appropriately and the patient's willing I feel like that usually can be avoided pretty easily.

Another participant discussed the importance of acknowledging and directly addressing this concern:

I think that any time you have somebody who has a new cancer recurrence and you're referring them to a different group of providers, you just worry, or the patient might worry, does this mean that you can't do anything else for me, does this mean that you're giving up on me, does this mean that you're just going to let me die, which you know certainly is not what it means when I refer people to palliative care, but feel like sometimes that needs to be very explicitly stated.

Participants also frequently discussed the value of normalizing palliative care referral to make it feel less threatening to patients: "I try to do that in an unobtrusive way by just making it kind of... what I do with all of my patients." One participant discussed both normalizing palliative care referral and adjusting the wording used to describe the service: "So, palliative care is something you do in the office in the same way you would have her see her internist for her diabetes.... I don't necessarily use the word palliative care; I use the term supportive care." Another participant explained how normalizing palliative care as just an additional layer of care increased patient acceptance of referral: "I mean, sometimes, if you talk of palliative care as just another layer of support, which is the catch phrase that I like to use, I find that very very few patients recoil from that."

\section{DISCUSSION}

Gynecologic oncologists at institutions with well-established palliative care services use a variety of strategies when presenting a palliative care referral to patients. These strategies aim to directly address the concern that patients may have negative associations with the idea of palliative care and thus may resist or decline referral.

Participants frequently focus initial palliative care referral on symptom management, even when symptoms are mild or there are additional reasons for referral such as discussion of goals of care or anticipated hospice needs. Symptom management is viewed as distinct from end-of-life issues, and this indication for referral may be less threatening to patients. When a patient accepts referral for symptom management, a relationship is developed with the palliative care team that promotes a smooth transition into end-of-life discussion and hospice should it be needed in the future. This is also an important strategy for situations in which the physician is uncomfortable or unwilling to discuss a poor prognosis. By providing an approach that can be used for patients with reservations about the full spectrum of palliative care services and by physicians without requiring a complete discussion of prognosis and goals, this strategy promotes physician adherence to national recommendations promoting the initiation of palliative care early in the disease course. 
Gynecologic oncologists also use patient trust, which arises from strong oncologist-patient relationships, to increase acceptance of palliative care referral. Patients are more likely to pursue palliative care referral if it is recommended by their oncologist. ${ }^{21}$ The unique longitudinal nature of gynecologic oncology, with the oncologist providing care from diagnosis, through surgery and chemotherapy, and into surveillance or end-of-life care, may be particularly important to a patient accepting the recommendation of palliative care. Developing strong oncologist-patient relationships and using patient trust to encourage acceptance of palliative care referral are strategies that promotes the integration of palliative care into standard oncology care.

Another strategy for overcoming negative patient views of palliative care is through clear explanation of what palliative care encompasses and normalization of early referral. Negative associations with the term palliative care have been well documented among both physicians ${ }^{22}$ and patients. ${ }^{23,24}$ There has been discussion about renaming palliative care; however, a recent study found that patients want a broader rebranding of palliative care and prefer an approach focusing on explanation, education, and incorporation into routine care..$^{25}$ Our study found that although physicians consider the possible negative connotations of the words palliative care and sometimes seek to use different terminology, they also believe that the most effective approach to addressing negative associations is through clear explanation and presenting referral as a routine part of care. This concordance between patients and physicians supports explanation and normalization as a promising strategy to facilitating acceptance of palliative care referral.

Strengths of our study include the large qualitative sample size and high response rate, as well as the inclusion of providers from multiple institutions with varying geography, size, and palliative care system structure. Because our study only included gynecologic oncologists at academic institutions with established outpatient palliative care clinics, our results may not be generalizable to medical, surgical, or radiation oncologists or to community practices or settings where palliative care is less available or less well established. Given that this study looks specifically at institutions with established palliative care access and integration, participants likely have an increased level of knowledge and awareness of palliative care services compared with gynecologic oncologists practicing in other settings and/or a predisposition to a positive view of palliative care integration into oncology. Although a positive bias toward palliative care may exist in the population surveyed, this provides an excellent starting point for discussion and for developing approaches to improving the integration of palliative care. In addition, the design of our study did not allow us to measure actual referral practices. A next step would be to directly assess the impact of these strategies on patient acceptance of palliative care referral and use of specialty palliative care services.

In summary, we found that gynecologic oncologists have specific strategies for introducing palliative care that proactively address negative patient associations and increase patient acceptance of referral. Strategies involve focusing initial referral on symptom management to encourage early relationship building and dissociate palliative care from end-oflife care, using trust built from strong oncologist-patient relationships, and normalizing and explaining palliative care services. These strategies provide groundwork for developing new approaches to improving the integration of palliative care into standard oncology care. JOP

\section{Acknowledgment}

Supported by National Center for Advancing Translational Sciences of the National Institutes of Health Award No. KL2TR000146 (Y.S.). The content of this article is solely the responsibility of the authors and does not necessarily represent the official views of the National Institutes of Health. Presented, in part, as a poster at the 2016 Palliative Care in Oncology Symposium, San Francisco, CA, September 9-10, 2016.

\section{Authors' Disclosures of Potential Conflicts of Interest}

Disclosures provided by the authors are available with this article at jop.ascopubs.org.

\section{Author Contributions}

Conception and design: Casey M. Hay, Carolyn Lefkowits, Yael Schenker Collection and assembly of data: Casey M. Hay, Marie A. Bakitas, Leslie H. Clark, Linda R. Duska, Renata R. Urban, Yael Schenker

Data analysis and interpretation: Casey M. Hay, Carolyn Lefkowits, Megan Crowley-Matoka, Marie A. Bakitas, Linda R. Duska, Stephanie L. Creasy, Yael Schenker

Manuscript writing: All authors

Final approval of manuscript: All authors

Accountable for all aspects of the work: All authors

Corresponding author: Casey M. Hay, MD, Division of Gynecologic Oncology, Department of Obstetrics, Gynecology and Reproductive Sciences, MageeWomen's Hospital of the University of Pittsburgh Medical Center, 300 Halket St, Pittsburgh, PA 15213; e-mail: haycm2@upmc.edu.

\section{References}

1. Choosing Wisely: Society of Gynecologic Oncology's Five Things Physicians and Patients Should Question. http://www.choosingwisely.org/societies/society-ofgynecologic-oncology/

2. Levy MH, Back A, Benedetti C, et al: NCCN clinical practice guidelines in oncology: Palliative care. J Natl Compr Canc Netw 7:436-473, 2009

3. Smith TJ, Temin S, Alesi ER, et al: American Society of Clinical Oncology provisional clinical opinion: The integration of palliative care into standard oncology care. J Clin Oncol 30:880-887, 2012 
4. Bakitas MA, Tosteson TD, Li Z, et al: Early versus delayed initiation of concurrent palliative oncology care: Patient outcomes in the ENABLE III randomized controlled trial. J Clin Oncol 33:1438-1445, 2015

5. Nevadunsky NS, Gordon S, Spoozak L, et al: The role and timing of palliative medicine consultation for women with gynecologic malignancies: Association with end of life interventions and direct hospital costs. Gynecol Oncol 132:3-7, 2014

6. Rugno FC, Paiva BS, Paiva CE: Early integration of palliative care facilitates the discontinuation of anticancer treatment in women with advanced breast or gynecologic cancers. Gynecol Oncol 135:249-254, 2014

7. Bakitas M, Lyons KD, Hegel MT, et al: Effects of a palliative care intervention on clinical outcomes in patients with advanced cancer: The Project ENABLE II randomized controlled trial. JAMA 302:741-749, 2009

8. Temel JS, Greer JA, Muzikansky A, et al: Early palliative care for patients with metastatic non-small-cell lung cancer. N Engl J Med 363:733-742, 2010

9. Zimmermann C, Swami N, Krzyzanowska M, et al: Early palliative care for patients with advanced cancer: A cluster-randomised controlled trial. Lancet 383:1721-1730 2014

10. Hui D, Elsayem A, De la Cruz M, et al: Availability and integration of palliative care at US cancer centers. JAMA 303:1054-1061, 2010

11. Hui D, Kim SH, Kwon JH, et al: Access to palliative care among patients treated at a comprehensive cancer center. Oncologist 17:1574-1580, 2012

12. Baile WF, Lenzi R, Parker PA, et al: Oncologists' attitudes toward and practices in giving bad news: An exploratory study. J Clin Oncol 20:2189-2196, 2002

13. Lopez-Acevedo M, Lowery WJ, Lowery AW, et al: Palliative and hospice care in gynecologic cancer: A review. Gynecol Oncol 131:215-221, 2013

14. Schenker Y, Crowley-Matoka M, Dohan D, et al: Oncologist factors that influence referrals to subspecialty palliative care clinics. J Oncol Pract 10: e37-e44, 2014
15. Smith CB, Nelson JE, Berman AR, et al: Lung cancer physicians' referral practices for palliative care consultation. Ann Oncol 23:382-387, 2012

16. Temel JS, McCannon J, Greer JA, et al: Aggressiveness of care in a prospective cohort of patients with advanced NSCLC. Cancer 113:826-833, 2008

17. Von Roenn JH, Voltz R, Serrie A: Barriers and approaches to the successful integration of palliative care and oncology practice. J Natl Compr Canc Netw 11: S11-S16, 2013 (suppl 1)

18. Hay CM, Lefkowits C, Crowley-Matoka M, et al: Gynecologic oncologist views influencing referral to outpatient specialty palliative care. Int J Gynecol Cancer 27: 588-596, 2017

19. Sandelowski M: Whatever happened to qualitative description? Res Nurs Health 23:334-340, 2000

20. Charmaz K: Constructing Grounded Theory. Thousand Oaks, CA, Sage Publications, 2006

21. Schenker Y, Park SY, Maciasz R, et al: Do patients with advanced cancer and unmet palliative care needs have an interest in receiving palliative care services? J Palliat Med 17:667-672, 2014

22. Fadul N, Elsayem A, Palmer JL, et al: Supportive versus palliative care: What's in a name? A survey of medical oncologists and midlevel providers at a comprehensive cancer center. Cancer 115:2013-2021, 2009

23. Center to Advance Palliative Care: 2011 Public Opinion Research on Palliative Care. https://media.capc.org/filer_public/3c/96/3c96a114-0c15-42da-a07f-11893cca7bf7/ 2011-public-opinion-research-on-palliative-care_237.pdf

24. Maciasz RM, Arnold RM, Chu E, et al: Does it matter what you call it? A randomized trial of language used to describe palliative care services. Support Care Cancer 21:3411-3419, 2013

25. Zimmermann C, Swami N, Krzyzanowska M, et al: Perceptions of palliative care among patients with advanced cancer and their caregivers. CMAJ 188:E217-E227, 2016 
Hay et al

AUTHORS' DISCLOSURES OF POTENTIAL CONFLICTS OF INTEREST

\section{Strategies for Introducing Outpatient Specialty Palliative Care in Gynecologic Oncology}

The following represents disclosure information provided by authors of this manuscript. All relationships are considered compensated. Relationships are self-held unless noted. I = Immediate Family Member, Inst = My Institution. Relationships may not relate to the subject matter of this manuscript. For more information about ASCO's conflict of interest policy, please refer to www.asco.org/rwc or ascopubs.org/journal/jop/site/misc/ifc.xhtml.

\section{Casey M. Hay}

No relationship to disclose

\section{Carolyn Lefkowits}

No relationship to disclose

\section{Megan Crowley-Matoka}

No relationship to disclose

\section{Marie A. Bakitas}

No relationship to disclose

\section{Leslie H. Clark}

No relationship to disclose

\section{Linda R. Duska}

Consulting or Advisory Role: Parexel, Advance Medical, Clearview Medical

Research Funding: Merck (Inst), Novartis (Inst), Pfizer (Inst), Tesaro (Inst), Abbott/AbbVie (Inst)

\section{Renata R. Urban}

No relationship to disclose

\section{Stephanie L. Creasy}

No relationship to disclose

\section{Yael Schenker}

No relationship to disclose 